\title{
FINE GRID ASTEROSEISMOLOGY OF G117-B15A AND R548
}

\author{
A. Bischoff-Kim, M. H. Montgomery, and D. E. Winget \\ Astronomy Department, University of Texas at Austin, 1 University Station, C1400, Austin, TX 78712; agnes@astro.as.utexas.edu, \\ mikemon@astro.as.utexas.edu, dew@astro.as.utexas.edu \\ Received 2007 July 11; accepted 2007 November 12
}

\begin{abstract}
We now have a good measurement of the cooling rate of G117-B15A. In the near future, we will have equally well determined cooling rates for other pulsating white dwarfs, including R548. The ability to measure their cooling rates offers us a unique way to study weakly interacting particles that would contribute to their cooling. Working toward that goal, we perform a careful asteroseismological analysis of G117-B15A and R548. We study them side by side because they have similar observed properties. We carry out a systematic, fine grid search for best-fit models to the observed period spectra of those stars. We freely vary four parameters: the effective temperature, the stellar mass, the helium layer mass, and the hydrogen layer mass. We identify and quantify a number of uncertainties associated with our models. Based on the results of that analysis and fits to the periods observed in R548 and G117-B15A, we clearly define the regions of the four-dimensional parameter space occupied by the best-fit models.
\end{abstract}

Subject headings: dense matter — stars: oscillations — stars: variables: other — white dwarfs

Online material: color figures

\section{ASTROPHYSICAL CONTEXT}

G117-B15A and R548 are pulsating white dwarfs with atmospheres dominated by hydrogen. These stars are called DAVs or ZZ Ceti stars. Their effective temperatures are close to $12,000 \mathrm{~K}$. They are nonradial, $g$-mode pulsators, in which the restoring force is buoyancy. Because of its potential use to study axions and other exotic physics (Isern et al. 1992; Kepler et al. 2005), G117-B15A was the object of several asteroseismological studies. R548 is similar to G117-B15A both spectroscopically and pulsationally, so studying them side by side may provide additional clues. Bradley (1998) performed such a parallel study. More recently Benvenuto et al. (2002) published an asteroseismological fit to G117-B15A's period spectrum. Benvenuto et al. evolved models from the main sequence and included detailed physics, such as time-dependent diffusion. Their best-fit model has an effective temperature and a mass consistent with spectroscopic results, but the periods of the model do not match the observed period spectrum as well as Bradley's fits.

We take a new approach to white dwarf asteroseismology, enabled by the computing resources available today. Using models similar to Bradley's, we perform a systematic, fine grid search for the best fits to the period spectra of G117-B15A and R548. We study both stars side by side. While we do not ignore the spectroscopy entirely, we take a naive approach and base much of our results on the period fitting alone. At the end we compare our results with the spectroscopy and previous studies. In the same naive spirit, we treat the helium layer mass as another free parameter. Bradley (1998) and Benvenuto et al. (2002) both fixed its value at $10^{-2} M_{*}$, where $M_{*}$ is the total mass of the model.

We organize our paper as follows. In $\S 2$ we present the observables we have for G117-B15A and R548. In $\S 3$ we summarize concisely the asteroseismological results of Benvenuto et al. (2002) and Bradley (1998). In $\S 4$ we explain our method. We include a discussion of our models and a quantitative study of what input parameters matter most. We present our results in $\S 5$, along with a discussion of how they compare with the fits done by Bradley (1998) and Benvenuto et al. (2002). We summarize and discuss our results in $\S 6$.

\section{THE STARS}

G117-B15A and R548 have much in common, and as we will see, studying them side by side helps constrain the model parameters. In Table 1 we list the spectroscopically determined effective temperatures and gravities of both stars, along with the modes we observe in those stars (Mukadam 2004). These are the clues we have at our disposal to find best-fit models to G117-B15A and R548.

The most solid observables listed in Table 1 are the periods. For both G117-B15A and R548, periods are determined to better than a second. The $215 \mathrm{~s}$ mode in G117-B15A and the $213 \mathrm{~s}$ mode in R548 are very stable and determined to extremely high accuracy. By comparing the amplitude of G117-B15A's 215 s mode in G117-B15A in the ultraviolet to those in the visible, Robinson et al. (1995) were able to determine confidently that it was an $\ell=1$ mode. R548's higher amplitude modes are close to the 215 and $271 \mathrm{~s}$ modes in G117-B15A and are likely $\ell=1$ modes as well.

Effective temperature and gravity are more poorly determined than the periods. We give an overview of the current spectroscopy in Table 1. To obtain an effective temperature for G117-B15A, Robinson et al. (1995) assumed a $\log g$ of 7.97, following work done by Daou et al. (1990).

\section{PREVIOUS FITS TO G117-B15A AND R548}

We summarize the best-fit models Benvenuto et al. (2002) found for G117-B15A in Table 2 and those Bradley (1998) found in Table $3 ; M_{\mathrm{H}}$ is the hydrogen layer mass, and $M_{\mathrm{He}}$ is the helium layer mass. We define $\Phi$ in equation (1). In essence, it is the average difference between the observed periods and the calculated periods. The lower the $\Phi$, the better the fit.

Both authors find two families of solutions: one in which the mode identification for G117-B15A's three observed periods is $k=1,2$, and 3 , and the other in which it is $k=2,3$, and $4(\ell=1)$. Bradley notes that for his models, both classes of solutions fit the observed periods and spectroscopic temperature equally well. Both authors find that the $k=1,2,3$ family of fits have thin hydrogen layers, and the $k=2,3,4$ family have thick hydrogen 
TABLE 1

Observed Properties of G117-B15A and R548

\begin{tabular}{|c|c|c|}
\hline Parameter & G117-B15A & R548 \\
\hline \multirow[t]{5}{*}{ 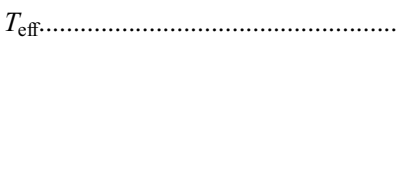 } & $11620 \pm 200$ & $11990 \pm 200(1)$ \\
\hline & $12830 \pm 350(2)$ & $\ldots$ \\
\hline & $12250 \pm 125(3)$ & $\ldots$ \\
\hline & $12010 \pm 180(4)$ & $11865 \pm 170$ \\
\hline & $12375 \pm 125(5)$ & $\ldots$ \\
\hline \multirow[t]{4}{*}{ 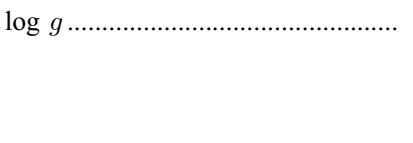 } & $7.97 \pm 0.05$ & $\ldots$ \\
\hline & $7.92 \pm 0.05(2)$ & $7.97 \pm 0.05(2)$ \\
\hline & $8.10 \pm 0.15$ & $\ldots$ \\
\hline & $7.94 \pm 0.17$ & $7.89 \pm 0.17$ \\
\hline \multirow[t]{6}{*}{ Periods (s) (amplitudes [mma]) ...... } & $215.22(19.8)$ & $212.77(4.1), 213.13(6.7)$ \\
\hline & $270.86(7.1)$ & $274.25(4.1), 274.78$ (2.9) \\
\hline & $304.15(8.8)$ & $\ldots$ \\
\hline & $\ldots$ & $187.27(1.0)$ \\
\hline & $\ldots$ & $318.08(0.9)$ \\
\hline & $\ldots$ & $333.65(1.0)$ \\
\hline
\end{tabular}

REFERENCES.-(1) Bergeron et al. 1995b; (2) Bergeron et al. 1995a; (3) Koester et al. 1994; (4) Koester \& Holberg 2001; (5) Robinson et al. 1995.

layers. Bradley's fits are hotter than Benvenuto et al.'s. Those are two trends to keep in mind.

Finally, we summarize the best-fit models Bradley found for R548 in Table 4. At the time, Bradley had at his disposal only two confirmed modes (the high-amplitude doublets near 213 and $274.5 \mathrm{~s}$ ) and one likely mode (the $318 \mathrm{~s}$ mode). Again, Bradley finds two classes of solutions: one with the three known modes identified as $k=1,2$, and 3, and the other as $k=2,3$, and 4 (all $\ell=1)$.

In Table 4 we have reevaluated $\Phi$ based on the knowledge that the $318 \mathrm{~s}$ mode is indeed real. Bradley could not rely on that mode and left it out. Including the $318 \mathrm{~s}$ mode, we immediately learn something new: the $k=2,3,4$ fits are significantly worse than the $k=1,2,3$ fits. If we do not include the 318 s mode, the two classes of fits are equally good. Bradley concludes that the $k=2,3,4$ fits are better because they agree with the spectroscopic temperatures better. He also computes the rotational splitting of the two modes and finds that they also appear to favor the $k=2,3,4$ fits.

\section{METHOD}

In essence, the problem we have to solve in white dwarf asteroseismology is the simple minimization of a function (the average difference between the calculated periods and the observed periods)

TABLE 2

Best Fits to G117-B15A's Observed Period Spectrum According to Benvenuto et al. (2002)

\begin{tabular}{|c|c|c|}
\hline Mode Identification & $k=1,2,3$ & $k=2,3,4^{\mathrm{a}}$ \\
\hline$T_{\mathrm{eff}}(\mathrm{K})$ & 11400 & 11800 \\
\hline$M_{\star} / M_{\odot} \ldots \ldots \ldots \ldots \ldots \ldots \ldots \ldots$ & 0.500 & 0.525 \\
\hline$M_{\mathrm{H}} / M_{*}$ & $10^{-6}$ & $1.48 \times 10^{-4}$ \\
\hline$M_{\mathrm{He}} / M_{*} \ldots \ldots$ & $10^{-2}$ & $10^{-2}$ \\
\hline$\Phi(\mathrm{s})$ & $\ldots$ & 6.5 \\
\hline
\end{tabular}

${ }^{a}$ Benvenuto et al. (2002) picked this fit because it matched the spectroscopy of Koester \& Allard (2000) better. Benvenuto et al. do not give a list of calculated periods for the $k=1,2,3 \mathrm{fit}$, but they show and state that it is comparable in quality to the $k=2,3,4$ fit. with $n$ variables, where $n$ is 4 in the present study. Those variables include $T_{\mathrm{eff}}, M_{*}, M_{\mathrm{He}}$, and $M_{\mathrm{H}}$. Expressed mathematically:

$$
\Phi\left(T_{\mathrm{eff}}, M_{*}, M_{\mathrm{He}}, M_{\mathrm{H}}\right)=\langle\Delta P\rangle=\frac{A}{N} \Sigma_{i=1}^{N}\left|P_{i}^{\mathrm{calc}}-P_{i}^{\mathrm{obs}}\right|,
$$

where $N$ is the number of observed periods and $A$ is a normalizing factor to account for the fact that at higher effective temperatures and higher masses, the period spacing decreases, artificially increasing our chances of finding a good period match. For the regions of parameter space considered in this work, $A \sim 1$.

The simplest way to minimize $\Phi$ is to compute it for all conceivable values of the four variables and pick the smallest value we find. But the number of times we have to evaluate $\Phi$ can quickly become astronomical. To make matters worse, each evaluation of the function requires the full computation of a white dwarf model. The White Dwarf Evolution Code (WDEC), described in $\S 4.1$, allows us to compute a large number of models in a small amount of time. But even with the WDEC, building a detailed map of $\Phi$ over all the relevant parameter space has only recently become practical on a standard desktop machine.

\subsection{Models}

The WDEC evolves hot polytrope models from temperatures close to $100,000 \mathrm{~K}$ down to the temperature of our choice. Models in the temperature range of interest for the present study are thermally relaxed solutions to the stellar structure equations. Each model we compute for the grid is the result of such an evolutionary sequence.

The WDEC is described in detail in Lamb \& van Horn (1975) and Wood (1990). We changed a few things since the work done by Bradley (1998). We used smoother core composition profiles and experimented with the more complex profiles that result from stellar evolution calculations (Salaris et al. 1997). We updated the envelope equation-of-state tables from those calculated by Fontaine et al. (1977) to those given by Saumon et al. (1995) and use OPAL opacities (Iglesias \& Rogers 1996). We also treated the abundance of elements differently at the boundary between the helium layer and the hydrogen layer. We assumed equilibrium diffusion profiles, following the prescription given by Arcoragi \& Fontaine (1980), but do not make the trace element approximation; 
TABLE 3

Best Fits to G117-B15A’s Observed Period Spectrum According to Bradley (1998)

\begin{tabular}{|c|c|c|c|c|c|c|}
\hline \multirow{2}{*}{$\frac{\text { Mode Identification }}{T_{\mathrm{eff}}(\mathrm{K}) \ldots \ldots \ldots \ldots \ldots \ldots \ldots}$} & \multicolumn{3}{|c|}{$k=1,2,3$} & \multicolumn{3}{|c|}{$k=2,3,4$} \\
\hline & 12160 & 11460 & 10790 & 12530 & 11860 & 11120 \\
\hline$M_{*} / M_{\odot} \ldots \ldots \ldots \ldots \ldots$ & 0.55 & 0.60 & 0.65 & 0.55 & 0.60 & 0.65 \\
\hline$M_{\mathrm{H}} / M_{*} \ldots \ldots \ldots \ldots$ & $3 \times 10^{-7}$ & $2 \times 10^{-7}$ & $1 \times 10^{-7}$ & $1.5 \times 10^{-4}$ & $8 \times 10^{-5}$ & $1 \times 10^{-5}$ \\
\hline$M_{\mathrm{He}} / M_{*} \ldots \ldots$ & $10^{-2}$ & $10^{-2}$ & $10^{-2}$ & $10^{-2}$ & $10^{-2}$ & $10^{-2}$ \\
\hline$\Phi(\mathrm{s})$ & 0.5 & 0.8 & 1.3 & 1.1 & 1.3 & 2.5 \\
\hline
\end{tabular}

this was shown to yield results quite similar to those based on time-dependent diffusion calculations (Althaus et al. 2003). We still treat diffusion at the carbon-helium transition zone as a free parameter.

There are three parameters associated with the shapes of the oxygen (and carbon) core composition profiles used by Bradley (1998): the central oxygen abundance $\left(X_{\mathrm{O}}\right)$, the edge of the homogeneous carbon and oxygen core $\left(q_{\mathrm{fm}}\right)$, and the point where the oxygen abundance drops down to zero $\left(q_{\mathrm{O}}\right) ; q$ is a mass coordinate, defined as $q(r)=-\log \left[1-M(r) / M_{*}\right]$, where $M(r)$ is the mass enclosed in radius $r$ and $M_{*}$ is the stellar mass. We show an example of a basic oxygen abundance profile and those three parameters in Figure 1, along with a Salaris-like profile. In Figure 2 we show abundance profiles for a fiducial model $(12,400 \mathrm{~K}$, $0.62 M_{\odot}, M_{\mathrm{He}}=10^{-2.3} M_{*}$, and $M_{\mathrm{H}}=10^{-7.7} M_{*}$ ), along with a model with a sharper carbon-helium transition zone. We will use both models in $\S 4.2$. Hereafter, the $M_{*}$ in the expression of the helium and hydrogen layer masses will be implicit. We adopted Salaris-like profiles for the carbon and oxygen abundances.

\subsection{The Parameters That Matter Most}

In order to determine the relative importance of each factor, we computed periods for the fiducial model we described above (one of our best-fit models for G117-B15A) and then varied one parameter at a time. Each time, we compared the resulting periods with the period spectrum of the fiducial model. In our analysis, we did not compare the full calculated period spectra, but instead picked the three periods that were closest to those found in the observed period spectrum of G117-B15A. This gives us an idea of what parameters matter most for fitting G117B15A and R548 in particular (although the same parameters in some order also matter for asteroseismological fits to other white dwarfs).

We list the results in order of decreasing significance in Table 5. In column (1) we list the properties of the fiducial model, in column (2) the changes we made in each case, and in column (3) the average difference between the periods of the fiducial model and the modified model (eq. [1], with $A=1$ ). We also detail the difference for each of the three periods of the fiducial model that match G117-B15A's observed period spectrum (216.3, 270.9, and $304.6 \mathrm{~s})$. The last two lines in Table 5 refer to the mixing-length theory (MLT) treatment of convection in the models; $\alpha$ is the ratio of the pressure scale height to the mixing length. ML2 refers to the prescription of Bohm \& Cassinelli (1971), and ML1 refers to that of Böhm-Vitense (1958). While convection is responsible for important nonlinear effects in the light curves (e.g., Montgomery 2005), it has very little effect on the periods of the modes excited in the models because it only affects the outer $10^{-12}$ mass fraction of the models or less.

Examination of how each change affects each mode individually reveals a few striking features. For instance, the $271 \mathrm{~s}$ mode is strongly affected by a change in the core parameter $X_{\mathrm{O}}$ $\left(\Delta P_{271 \mathrm{~s}}=7.18 \mathrm{~s}\right)$, while the other two modes are not $\left(\Delta P_{216 \mathrm{~s}}=\right.$ 0.58 and $\left.\Delta P_{305 \mathrm{~s}}=1.69 \mathrm{~s}\right)$. This, and other features apparent in the columns (4)-(6) of Table 5, can be explained by examining the weight functions of the relevant modes. Montgomery et al. (2003) used them as a diagnostic of the effect of composition transition zones on mode trapping. In Figure 3 we show the weight functions of the first three $\ell=1$ modes for the fiducial model of Table 5. In the second panel of Figure 3, we see that the $k=1$ mode resonates strongly with the base of the He layer. This shows that it is extremely sensitive to the He layer mass, and very insensitive to the $\mathrm{H}$ layer mass, which is what we see from $\Delta P_{216} \mathrm{~s}$ in Table 5. Looking at the third panel, we note that the $k=2$ mode predominantly samples the chemical profile in the core; its period change due to a change in $\mathrm{C} / \mathrm{O}$ profile is 5 times that of the other two modes. This explains why the $271 \mathrm{~s}$ mode is so sensitive to the core parameters. The last panel shows that the $k=3$ mode samples both of these features, as well as farther out in the model near the base of the $\mathrm{H}$ layer. This mode is the most sensitive of the three to the H layer mass.

In order to keep our model grids manageable, we decided to vary four parameters. While $T_{\mathrm{eff}}, M_{*}, M_{\mathrm{H}}$, and $M_{\mathrm{He}}$ have by far the largest effect on the periods, the parameters associated with the core abundance profiles and the shape of the carbon-helium transition zone (second block in Table 5) can have a significant effect (as high as $\sim 3 \mathrm{~s}$ ) on the pulsation periods. Since we had to fix our core composition profiles, we decided to fix them to profiles predicted by stellar evolution (Fig. 2). We also adopted the fiducial model's carbon-helium transition profiles.

In the asteroseismological fits detailed in $\S 5$ we use the periods rounded to the second, and do not distinguish a model period that fit within $0.5 \mathrm{~s}$ from one that fits within $1 \mathrm{~s}$. This allows

TABLE 4

Best Fits to R548's Observed Period Spectrum According to Bradley (1998)

\begin{tabular}{|c|c|c|c|c|c|c|}
\hline \multirow{2}{*}{$\frac{\text { Mode Identification }}{T_{\text {eff }}(\mathrm{K}) \ldots \ldots \ldots \ldots \ldots \ldots \ldots \ldots \ldots \ldots \ldots \ldots \ldots \ldots \ldots \ldots}$} & \multicolumn{3}{|c|}{$k=1,2,3$} & \multicolumn{3}{|c|}{$k=2,3,4$} \\
\hline & 12440 & 11560 & 10790 & 12190 & 11970 & 11320 \\
\hline$M_{*} / M_{\odot} \ldots \ldots \ldots \ldots \ldots \ldots \ldots \ldots \ldots \ldots$ & 0.55 & 0.60 & 0.65 & 0.55 & 0.60 & 0.65 \\
\hline$M_{\mathrm{H}} / M_{*} \ldots \ldots \ldots \ldots$ & $3 \times 10^{-7}$ & $1 \times 10^{-7}$ & $5 \times 10^{-8}$ & $1.5 \times 10^{-4}$ & $5 \times 10^{-5}$ & $5 \times 10^{-6}$ \\
\hline 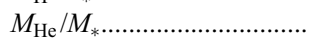 & $10^{-2}$ & $10^{-2}$ & $10^{-2}$ & $10^{-2}$ & $10^{-2}$ & $10^{-2}$ \\
\hline$\Phi(\mathrm{s})$ & 3.2 & 1.3 & 0.3 & 4.4 & 4.6 & 3.1 \\
\hline
\end{tabular}




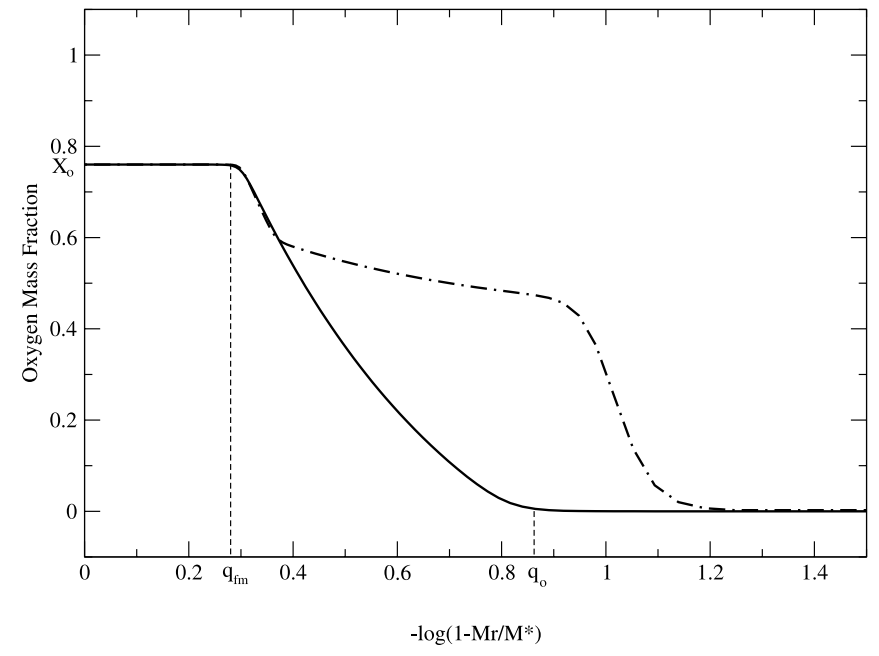

FIg. 1.-Basic oxygen abundance profile (solid curve) and the three associated parameters $X_{\mathrm{O}}, X_{\mathrm{fm}}$, and $q_{\mathrm{O}}$. The dash-dotted curve is an example of a Salaris-like oxygen abundance profile.

us not to worry ourselves with the factors listed in the last block of Table 5 and other possible small effects.

\subsection{Fine Grid Search}

We started by building a low-resolution grid that covered a broad region of parameter space, guided by the spectroscopy ( Table 1). We varied masses between 0.46 and $0.80 M_{\odot}$ and temperatures between 10,800 and $13,000 \mathrm{~K}$. We considered $-3<$ $\log \left(M_{\mathrm{He}}\right)<-2$ and $-8.4<\log \left(M_{\mathrm{H}}\right)<\log \left(M_{\mathrm{He}}\right)-2$. For that grid, we determined that step sizes of $200 \mathrm{~K}$ in $T_{\text {eff }}, 0.02 M_{\odot}$ in $M_{*}$, and 0.4 in $\log \left(M_{\mathrm{He}}\right)$ and $\log \left(M_{\mathrm{H}}\right)$ were sufficient to locate likely minima of $\Phi$. Using the results of the broad grid search, we narrowed down our search to smaller areas of parameter space and built a finer grid. We found that a resolution of $100 \mathrm{~K}$ in $T_{\text {eff }}$, $0.01 M_{\odot}$ in $M_{*}$, and 0.2 in $\log \left(M_{\mathrm{He}}\right)$ and $\log \left(M_{\mathrm{H}}\right)$ was sufficient to clearly define the minima of $\Phi$. The broad grid contains nearly 20,000 models; the fine grid contains 6000 (it is more limited in parameter space).

For G117-B15A, we further narrowed down the list of possible best fits by assuming that G117-B15A's modes were $\ell=1$ modes, consistent with mode identification work done by Robinson et al. (1995) and the fact that higher 1 modes suffer from geometric cancellation at the surface of the star and are likely to result in low-amplitude pulsations. For R548, we required that the two highamplitude modes, which also seem to be present in G117-B15A (see Table 1), be $\ell=1$ modes. We did not place any constraints on the identification of the other three modes.

\section{RESULTS}

\subsection{Mass and Effective Temperature}

We display the results of the fine grid search for G117-B15A and R548 side by side in the $M_{*}-T_{\text {eff }}$ plane in Figure 4 . We explored all of the parameter space shown. The fine grid starts at $0.6 M_{\odot}$ and above. We also indicate the spectroscopic temperature determinations for both stars, as well as masses derived from the gravities listed in Table 1, using our models. Our best-fit models appear systematically massive and/or hot compared to the spectroscopic results. We will come back to this discrepancy in $\S 6$.

With our $1 \mathrm{~s}$ sensitivity cutoff due to small modeling uncertainties, we are unable to determine a unique point in parameter

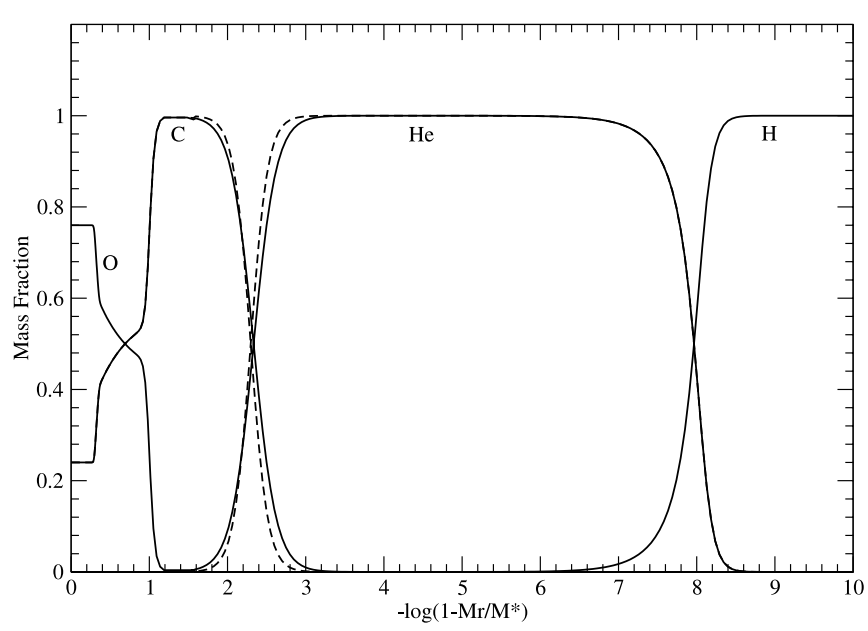

FIG. 2.-Chemical composition profiles for the fiducial model (solid lines) and for a model with a sharper carbon-helium transition zone (dashed lines). [See the electronic edition of the Journal for a color version of this figure.]

space that matches G117-B15A or R548. Instead, we find families of solutions. There is a tight correlation between the mass and the effective temperature, anticipated from earlier work (Bradley 1998). Decreasing either the temperature or the mass decreases the spatial average of the Brunt-Väisälä frequency in a similar way, and therefore yields similar sets of periods. In general, our best-fit models are more massive and/or hotter than we would have expected from the spectroscopy.

From their observed properties (Table 1), we expected G117B15A and R548 to be best fit by similar models. We do see that in our results. It also comes as no surprise that the $\ell=1$ identification requirement does not limit model fits to R548 as much as they do for G117-B15A. To obtain the dotted circles in Figure 4, we discarded fits that did not obey the $\ell=1$ mode identification for G117-B15A's three modes and for R548's two high-amplitude modes (regardless of the quality of the fit). Because this first cut is based on the constraint of a fewer number of modes in the case of R548, it does not eliminate as many models. On the other hand, we have three additional modes to fit for R548, and they ultimately allow us to narrow down the best-fit models to a small region of parameter space. We do not have that luxury with G117$\mathrm{B} 15 \mathrm{~A}$, as the three $\ell=1$ modes are all we have. We also obtain better fits to G117-B15A's three periods than we do to R548's five periods, as we would expect.

\subsection{Helium and Hydrogen Layer Mass}

For both stars, the helium layer mass appears to be fairly well constrained around $4 \times 10^{-3}$. It is determined by the $215 \mathrm{~s}$ mode in G117-B15A and the 213 s mode in R548. If we do not include those modes in the fit, $M_{\mathrm{He}}$ is essentially unconstrained. For G117B15A, if we change that mode by as little as $5 \mathrm{~s}$ (e.g., 215-210 s), the helium layer mass changes from $4 \times 10^{-3}$ to $5 \times 10^{-3}$. If instead we leave out the $271 \mathrm{~s}$ mode, the helium layer mass remains constrained. This result is readily understandable from Figure 3 and the associated discussion of weight functions in $\S 4.2$.

In Figure 5 we show the very best fit models $(\Phi \leq 1 \mathrm{~s}$ for G117-B15A and $\Phi \leq 1.5 \mathrm{~s}$ for R548) in the $M_{*}-T_{\text {eff }}$ plane and indicate their respective hydrogen layer masses. We discover that families of models with different hydrogen layer masses separate out in the $M_{*}-T_{\text {eff }}$ plane.

We find that R548 is best fit with thin hydrogen layer models $\left[M_{\mathrm{H}} \simeq(2-6) \times 10^{-8}\right]$. For G117-B15A, we find two well-defined 
TABLE 5

Parameters That Matter Most for DAV Models

\begin{tabular}{|c|c|c|c|c|c|}
\hline $\begin{array}{c}\text { Fiducial Model } \\
\text { (1) }\end{array}$ & $\begin{array}{l}\text { Change } \\
\text { (2) }\end{array}$ & $\begin{array}{c}\langle\Delta P\rangle \\
\text { (s) } \\
(3)\end{array}$ & $\begin{array}{c}\Delta P_{216} \\
\text { (s) } \\
(4)\end{array}$ & $\begin{array}{c}\Delta P_{271} \\
\quad(\mathrm{~s}) \\
(5)\end{array}$ & $\begin{array}{c}\Delta P_{305} \\
\text { (s) } \\
(6)\end{array}$ \\
\hline$M_{*}=0.62 M_{\odot} \ldots \ldots \ldots \ldots \ldots$ & $M_{*}=0.56 M_{\odot}(-10 \%) \ldots \ldots \ldots \ldots$ & 16.7 & +10.1 & +18.1 & +22.0 \\
\hline Salaris profiles..................... & Basic profiles .................................... & 15.2 & -7.40 & -32.3 & -6.01 \\
\hline$T_{\text {eff }}=12,400 \mathrm{~K} \ldots \ldots \ldots \ldots$ & $T_{\text {eff }}=11,160 \mathrm{~K}(-10 \%) \ldots \ldots \ldots \ldots$ & 12.9 & +12.1 & +15.4 & +11.3 \\
\hline$M_{\mathrm{H}}=10^{-7.7} \ldots \ldots \ldots \ldots \ldots \ldots$ & $M_{\mathrm{H}}=10^{-6.9}(-10 \%$ in $\log ) \ldots \ldots$ & 4.59 & -0.273 & +3.29 & +10.2 \\
\hline$M_{\mathrm{He}}=10^{-2.3} \ldots \ldots \ldots \ldots \ldots$ & $M_{\mathrm{He}}=10^{-2.1}(-10 \%$ in $\log ) \ldots$. & 3.88 & -5.19 & +1.95 & -4.50 \\
\hline$X_{\mathrm{O}}=0.76 \ldots \ldots \ldots \ldots$ & $X_{\mathrm{O}}=0.68(-10 \%) \ldots \ldots \ldots \ldots$ & 3.15 & +0.58 & +7.18 & +1.69 \\
\hline Soft $\mathrm{C} / \mathrm{He}$ transition ....... & Sharper $\mathrm{C} / \mathrm{He}$ transition................... & 2.61 & -4.28 & +0.713 & -2.84 \\
\hline$q_{\mathrm{fm}}=0.50 \ldots \ldots \ldots \ldots \ldots \ldots$ & $q_{\mathrm{fm}}=0.45(-10 \%)$ & 1.18 & +0.0439 & -2.70 & -0.791 \\
\hline Saumon et al. (1995) ...... & Fontaine et al. (1977) .................. & 1.08 & $\ldots$ & $\ldots$ & $\ldots$ \\
\hline Envelope EOS..................... & 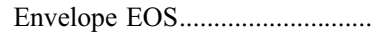 & $\ldots$ & $\ldots$ & $\ldots$ & $\ldots$ \\
\hline$\alpha=0.6 \ldots \ldots \ldots \ldots \ldots \ldots \ldots \ldots \ldots$ & $\alpha=2$ & 0.0104 & $\ldots$ & $\ldots$ & $\ldots$ \\
\hline ML2 & ML1 & 0.0000689 & $\ldots$ & $\ldots$ & $\ldots$ \\
\hline
\end{tabular}

families: one at higher mass and lower effective temperature with $M_{\mathrm{H}}=6.3 \times 10^{-7}$, and one at lower mass and higher effective temperature with $M_{\mathrm{H}} \simeq(1-4) \times 10^{-8}$. Previous investigations have found both thick and thin solutions (Bradley 1998; Benvenuto et al. 2002). Based on the incompleteness of our current understanding of mass loss in the late stages of stellar evolution, we do not believe either set of models can be ruled out by stellar evolution calculations. For instance, Althaus et al. (2002) present the hydrogen abundance $\left(M_{\mathrm{H}}=10^{-4}\right)$ in DAVs as an upper limit, as it would be reduced by the inclusion of mass loss episodes during the planetary phase, the extent of which is unknown. While none of our best fits truly have thick hydrogen layers, we will refer to the two distinct families as "thick" and "thin" hydrogen layer fits.

\subsection{Mode Identification}

Previous asteroseismological studies of G117-B15A (Bradley 1998; Benvenuto et al. 2002) identified the three modes as consecutive $\ell=1$ modes, with the $215 \mathrm{~s}$ mode being either a $k=1$ or a $k=2$ mode. Bradley found equally good fits with either mode identification. While Benvenuto et al.'s best-fit model was consistent with a $k=1,2,3$ mode identification, they also found good fit models with $k=2,3,4$ modes. Among the 27 models in the fine grid that matched the observed periods to better than $1 \mathrm{~s}$ on the average, we found 13 that matched the observed periods with $k=1,2,3$ modes and 14 with $k=2,3,4$ modes. The former family of models all have $M_{\mathrm{H}} \simeq(1-4) \times 10^{-8}$, and the latter have $M_{\mathrm{H}}=6.3 \times 10^{-7}$. This is qualitatively in tune with what Bradley (1998) and Benvenuto et al. (2002) found (the $k=1,2,3$ fits have thin hydrogen layers, and the $k=2,3,4$ fits have thicker hydrogen layers).

For R548, among the top 20 best-fit models, we find that the most likely mode identification (17 out of 20 models) is $\ell=1, k=1,2,4$, respectively, for the $213,274.5$, and $318 \mathrm{~s}$ modes, and $\ell=2, k=4$ and 8 for the 187 and 334 s modes. Recall that we required the 213 and $274.5 \mathrm{~s}$ modes to be $\ell=1$ modes, but did not place any constraints on the other modes. Two out of the three models that disagree with this mode identification are thick hydrogen models (while the 17 models with the most common mode identification are thin hydrogen models). The $\ell=2, k=4$ identification for the $187 \mathrm{~s}$ mode is very robust $(20 / 20)$.

The main reason why we are studying G117-B15A and R548 side by side is that they are observationally similar, and we therefore expect them to also be structurally similar. If that is the case, then the mode identification results for R548 suggest that the correct mode identification for G117-B15A is $k=1,2$, and 3 , in favor of the thin hydrogen layer and lower stellar mass solutions $\left[M_{\mathrm{H}} \simeq(1-4) \times 10^{-8}\right]$.

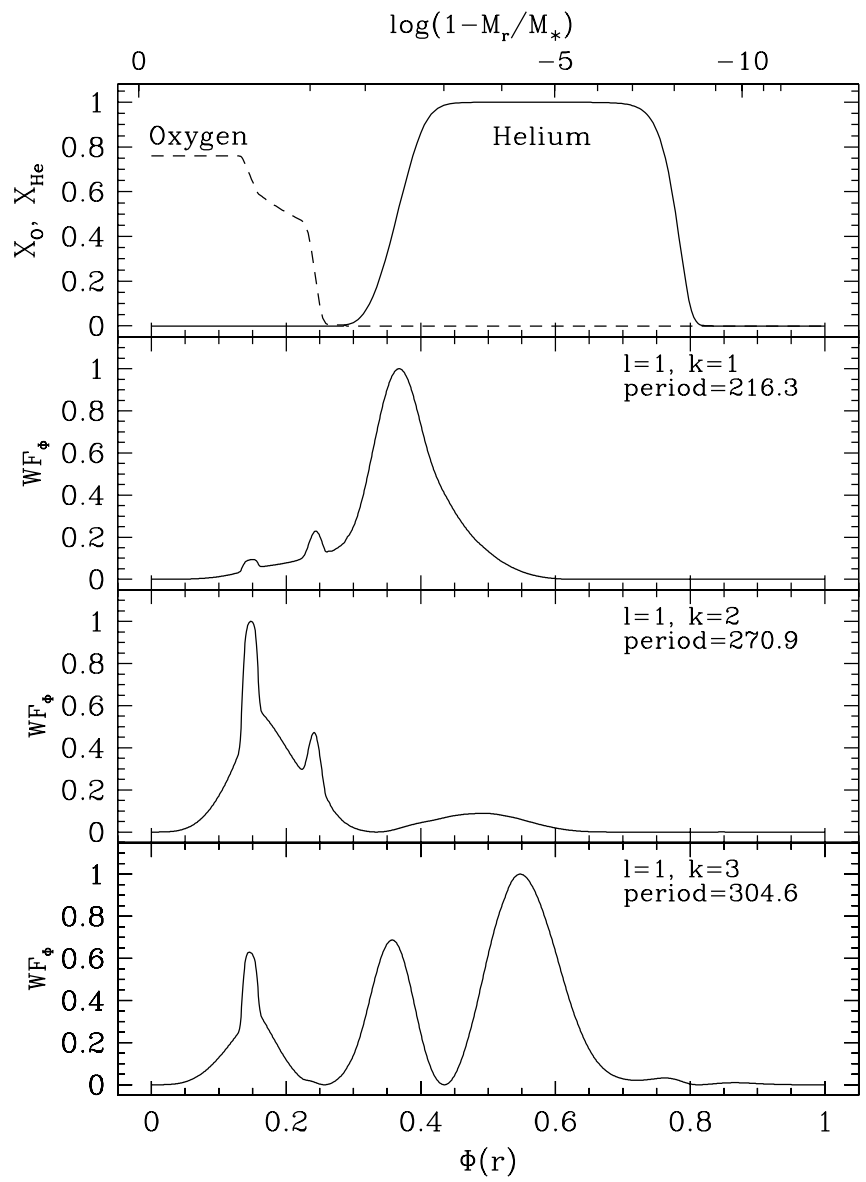

FIG. 3.-Plot of the weight functions of the first three $\ell=1$ overtones as a function of the coordinate $\Phi(r)$, the "normalized buoyancy radius," for the fiducial model of Table 5 . We see that the $k=1$ mode has its period mainly determined by the composition gradient at the base of the He layer, and that the $k=2$ mode is most sensitive to the structure in the $\mathrm{C} / \mathrm{O}$ profile in the core. The $k=3$ mode is sensitive to multiple features in the model and the only one sensitive to the location of the base of the hydrogen layer. [See the electronic edition of the Journal for a color version of this figure.] 


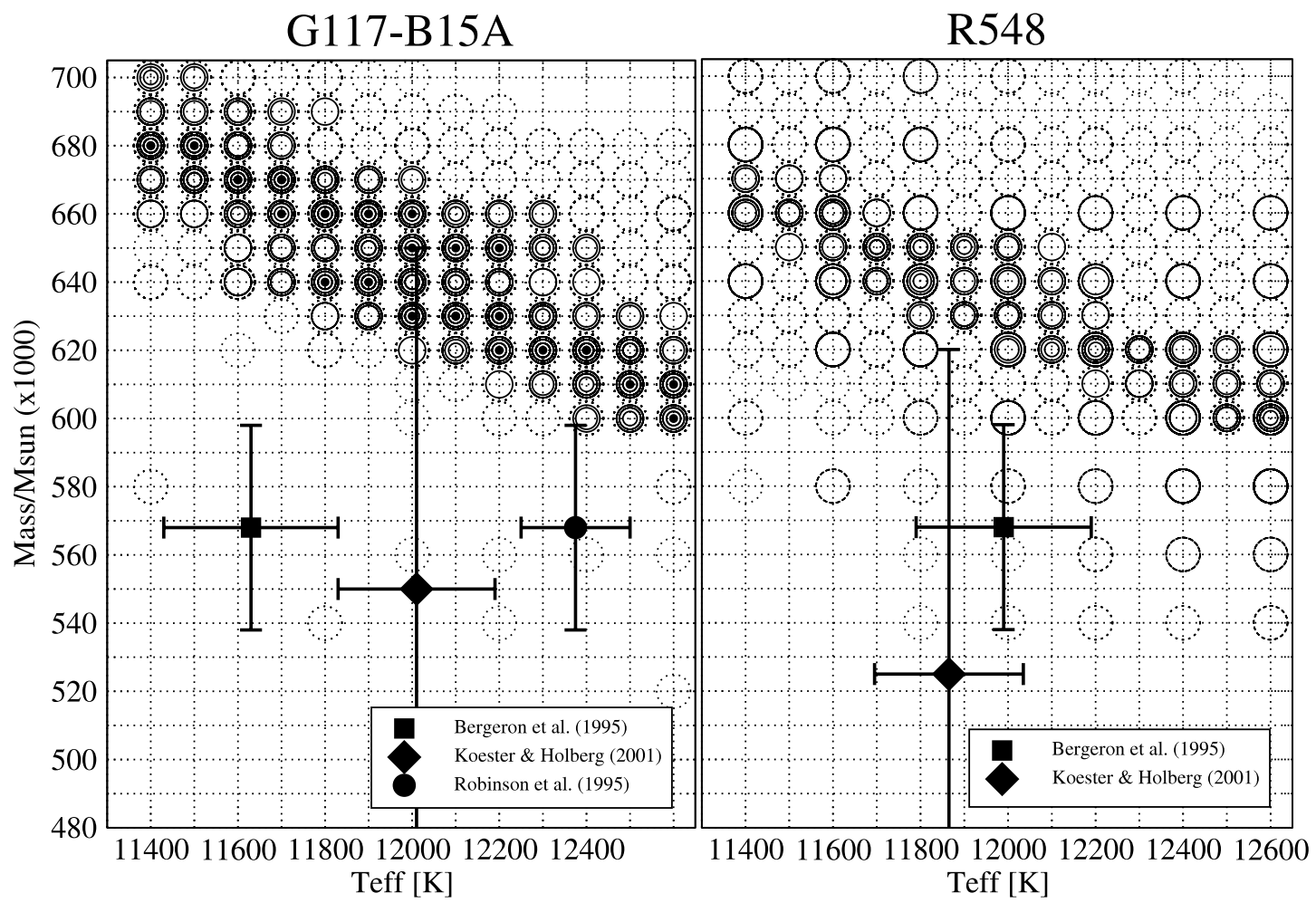

Fig. 4.-Valley of best-fit models for G117-B15A and R548 in the $M_{\star}-T_{\text {eff }}$ plane. The dashed circles mark the location of the subset of models that fit the $\ell=1$ mode identification criterion (see text). Of those, the progressively filled circles indicate better and better fits $(\Phi<2.5,2,1.5$, and $1 \mathrm{~s}$, respectively). The symbols with error bars indicate the spectroscopically determined temperatures and mass for G117-B15A and R548, according to the legend. We used our models to derive a mass from the gravities listed in Table 1. [See the electronic edition of the Journal for a color version of this figure.]

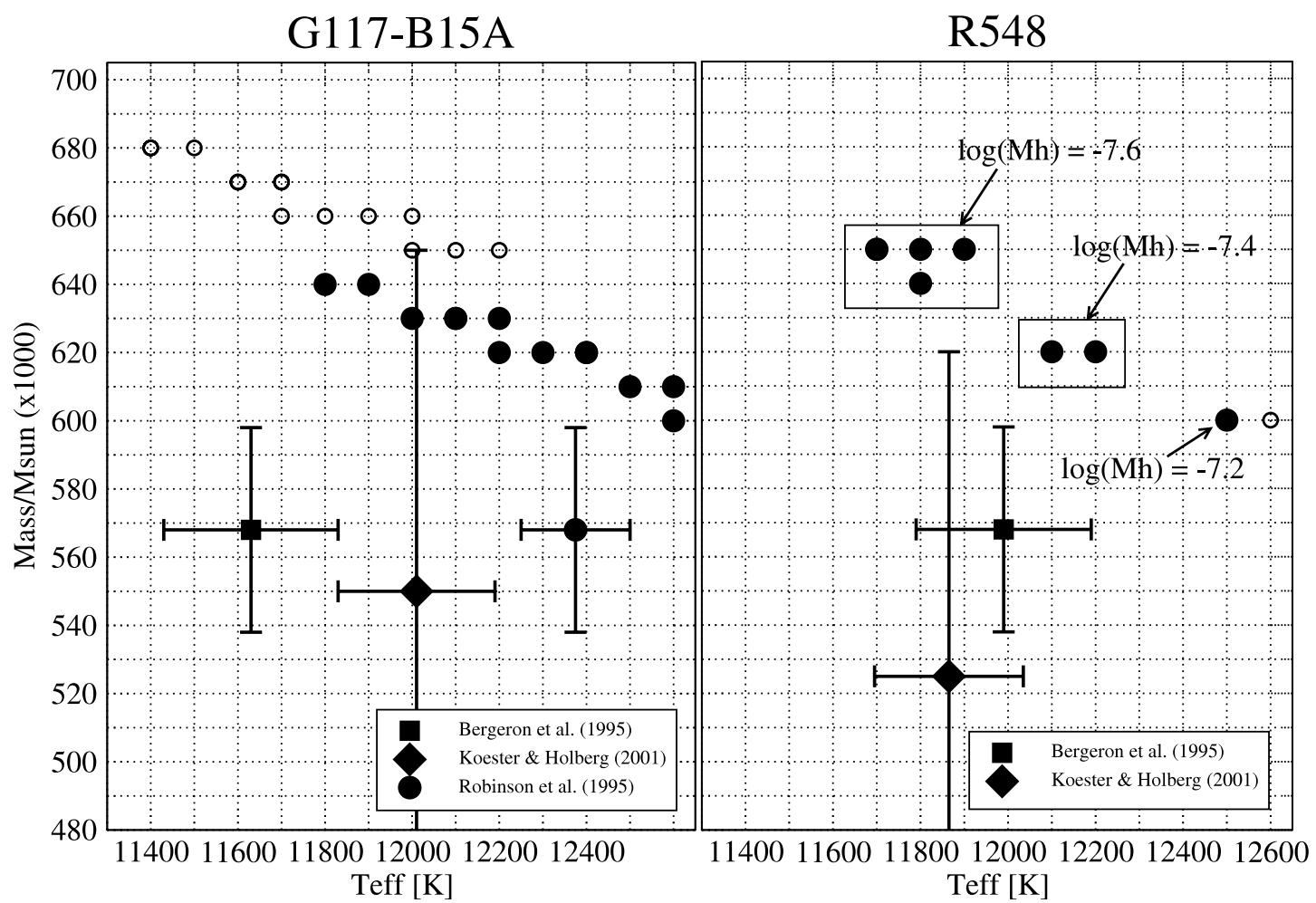

FIG. 5. - Best-fit models in the $M_{*}-T_{\text {eff }}$ plane for different hydrogen layer masses. For G117-B15A, we isolated the solutions for which $\Phi<1 \mathrm{~s}$, and for R548, those for which $\Phi<1.5 \mathrm{~s}$. The open circles correspond to "thick" hydrogen solutions $\left(M_{\mathrm{H}}=6.3 \times 10^{-7}\right)$, while the filled circles correspond to thin hydrogen solutions $\left[M_{\mathrm{H}} \simeq(1-4) \times 10^{-8}\right]$. [See the electronic edition of the Journal for a color version of this figure.] 


\section{SUMMARY AND CONCLUSIONS}

We performed a systematic fine grid search for best-fit models to the two DAVs G117-B15A and R548. We find best-fit models for both stars between 11,600 and $12,700 \mathrm{~K}$ and between 0.59 and $0.66 M_{\odot}$. In both cases, the region occupied by the best-fit models in the $M_{*}-T_{\text {eff }}$ plane is related to the thickness of the hydrogen layer. Treating the helium layer mass as a free parameter, we discovered that the lowest period mode for each star $(215 \mathrm{~s}$ for G117-B15A and 213 s for R548) single-handedly sets the helium layer mass to $4 \times 10^{-3}$. Both stars are well fit with thin hydrogen layer models $\left(10^{-7}<M_{\mathrm{H}}<10^{-8}\right)$. For G117-B15A, we find a second family of solutions between 11,300 and 12,300 $\mathrm{K}$ and between 0.65 and $0.68 M_{\odot}$. Those fits have slightly thicker hydrogen layers $\left(M_{\mathrm{H}}=6.3 \times 10^{-7}\right)$.

For R548 we find a unique, robust mode identification. The dominant modes (213 and $274.5 \mathrm{~s}$ ) are $\ell=1, k=1,2$ modes. The $318 \mathrm{~s}$ mode is also an $\ell=1$ mode, with $k=4$. The last two modes (187 and $334 \mathrm{~s}$ ) are $\ell=2, k=4$ and 8 , respectively. For G117-B15A, we find two distinct families of best-fit models. Models with $M_{\mathrm{H}}=6.3 \times 10^{-7}$ all have the same mode identification, namely $k=2,3$, and 4 for the three observed periods (215, 271 , and $304 \mathrm{~s})$. Models with $M_{\mathrm{H}} \simeq(1-4) \times 10^{-8}$ are consistent with G117-B15A's periods being $k=1,2$, and 3. R548 and G117B15A have similar observed properties, and based on that fact, it is likely that they have similar structures. In this case, the second class of models appears better, but we cannot discard the first class of models based on that fact alone.

By sampling parameter space systematically and homogeneously, we found that our models were offset in mass and temperature compared to the spectroscopy (the models are hotter and/or more massive). Reid (1996) measured the gravitational redshift of spectral lines for G117-B15A. The resulting mass is in tune with the spectroscopic mass $\left(0.536 \pm 0.010 M_{\odot}\right)$. Both different equationof-state tables and opacities could alter the structure of the models and possibly modify our results. We do not expect either to have a large effect, however. Just recently, Cassisi et al. (2007) published new electron-conduction opacities that treat the partially degenerate regime relevant in white dwarf envelopes better. According to Cassisi et al., the new opacities differ only by at most a factor of 2 in white dwarf envelopes and have very little effect on the mass-radius relation. We also tried to revert back to the Fontaine et al. (1977) envelope equations of state and found that this had a negligible effect on the gravities in our models.

Results from $\S 4.2$ suggest a more promising avenue. They show that structure in the core and the shape of the helium composition profile at the carbon-helium interface can have a large effect on the pulsation periods of a model. We adopted core composition profiles from stellar evolution calculations and did not vary them. Our asteroseismological results suggest that perhaps we should try different core composition profiles. With precise enough determinations of G117-B15A and R548's mass and effective temperature from spectroscopy or other independent methods, one can turn the problem around and attempt to determine what composition profiles are needed in order to reconcile the asteroseismology with the spectroscopy. While preliminary results appear promising, a full investigation requires the clever analysis of a more extensive grid of models, and we leave that for a future publication.

Once we are satisfied that we have models that match both asteroseismologically and spectroscopically, we can calculate $\dot{P}$ values for our models and use them together with the observed $\dot{P}$ values to constrain the energy loss rate due to any weakly interacting particles, such as axions. The fine grid approach allows us to formally assess the uncertainties in our models' parameters and to obtain tight constraints on the emission rate of weakly interacting particles in G117-B15A.

We wish to thank our referee for helping us improve on our work and giving us new ideas. We thank E. Robinson for providing essential feedback. This work was made faster and easier thanks to the neatly packaged code provided by T. Metcalfe. This research was supported by NSF grant AST 05-07639.
Althaus, L. G., Serenelli, A. M., Córsico, A. H., \& Benvenuto, O. G. 2002, MNRAS, 330, 685

Althaus, L. G., Serenelli, A. M., Córsico, A. H., \& Montgomery, M. H. 2003, A\&A, 404, 593

Arcoragi, J.-P., \& Fontaine, G. 1980, ApJ, 242, 1208

Benvenuto, O. G., Córsico, A. H., Althaus, L. G., \& Serenelli, A. M. 2002, MNRAS, 332, 399

Bergeron, P., Liebert, J., \& Fulbright, M. S. 1995b, ApJ, 444, 810

Bergeron, P., Wesemael, F., Lamontagne, R., Fontaine, G., Saffer, R. A., \& Allard, N. F. 1995a, ApJ, 449, 258

Bohm, K. H., \& Cassinelli, J. 1971, A\&A, 12, 21

Böhm-Vitense, E. 1958, Z. Astrophys., 46, 108

Bradley, P. A. 1998, ApJS, 116, 307

Cassisi, S., Potekhin, A. Y., Pietrinferni, A., Catelan, M., \& Salaris, M. 2007, ApJ, 661, 1094

Daou, D., Wesemael, F., Fontaine, G., Bergeron, P., \& Holberg, J. B. 1990, ApJ, 364, 242

Fontaine, G., Graboske, H. C., Jr., \& van Horn, H. M. 1977, ApJS, 35, 293

Iglesias, C. A., \& Rogers, F. J. 1996, ApJ, 464, 943

\section{REFERENCES}

Isern, J., Hernanz, M., \& Garcia-Berro, E. 1992, ApJ, 392, L23

Kepler, S. O., Costa, J. E. S., Mukadam, A., Mullally, F., Winget, D. E., Nather, R. E., \& Sullivan, D. 2005, in ASP Conf. Ser. 334, 14th European Workshop on White Dwarfs, ed. D. Koester \& S. Moehler (San Francisco: ASP), 501 Koester, D., \& Allard, N. F. 2000, Baltic Astron., 9, 119

Koester, D., Allard, N. F., \& Vauclair, G. 1994, A\&A, 291, L9

Koester, D., \& Holberg, J. B. 2001, in ASP Conf. Ser. 226, 12th European Workshop on White Dwarfs, ed. J. L. Provencal et al. (San Francisco: ASP), 299

Lamb, D. Q., \& van Horn, H. M. 1975, ApJ, 200, 306

Montgomery, M. H. 2005, ApJ, 633, 1142

Montgomery, M. H., Metcalfe, T. S., \& Winget, D. E. 2003, MNRAS, 344, 657

Mukadam, A. S. 2004, Ph.D. thesis, Univ. Texas, Austin

Reid, I. N. 1996, AJ, 111, 2000

Robinson, E. L., et al. 1995, ApJ, 438, 908

Salaris, M., Dominguez, I., Garcia-Berro, E., Hernanz, M., Isern, J., \& Mochkovitch, R. 1997, ApJ, 486, 413

Saumon, D., Chabrier, G., \& van Horn, H. M. 1995, ApJS, 99, 713

Wood, M. A. 1990, Ph.D. thesis, Univ. Texas, Austin 